$7-2020$

\title{
Acute syphilitic posterior placoid chorioretinopathy: A case report
}

Rehman Siddiqui

Syed Zohaib Maroof Hussain

Irfan Jeeva

Raeefuddin Ahmed

Follow this and additional works at: https://ecommons.aku.edu/pakistan_fhs_mc_surg_ophthalmol

Part of the Eye Diseases Commons, Ophthalmology Commons, and the Surgery Commons 


\section{Acute syphilitic posterior placoid chorioretinopathy: A case report}

M. A. Rehman Siddiqui,, ${ }^{1}$ Syed Zohaib Maroof Hussain, ${ }^{2}$ Irfan Jeeva, ${ }^{3}$ Raeefuddin Ahmed 4

\begin{abstract}
A 25-year-old male presented with a complaint of acute visual loss in his right eye for two days. There was a history of visual loss in the left eye six months ago. Comprehensive eye examination showed evidence of posterior uveitis, and a placoid lesion on the right fundus. Further physical examination showed maculopapular rashes on the upper back which was highly suggestive of syphilis. Blood tests for venereal disease research laboratory (VDRL) test and fluorescent treponemal antibody absorption (FTA-ABS) test were ordered, which were both positive. Ocular syphilis should be suspected in all cases of uveitis. Early diagnosis and prompt treatment with appropriate antibiotics can prevent permanent visual loss.
\end{abstract}

Keywords: Syphilis, chorioretinitis and uveitis.

\section{DOl: https://doi.org/10.5455/JPMA.35876}

\section{Introduction}

Syphilis is a multi-systemic disease and is known as the "great imitator". Diagnosis of syphilis is challenging, particularly of ocular syphilis. There are no pathognomonic diagnostic signs of ocular syphilis. Syphilis can involve any part of the eye at any stage of the disease. ${ }^{1}$ Ocular syphilis commonly presents as uveitis or interstitial keratitis. ${ }^{2}$ Vision may be permanently affected if the diagnosis and prompt treatment is delayed.1,3 There is a recent increase in the number of syphilis cases reported worldwide.1,3 Between 1999 and 2004, a 15-fold rise in incidence of ocular syphilis has been reported in the United Kingdom. ${ }^{3}$ To the best of our knowledge, acute syphilitic placoid posterior chorioretinopathy has not been described previously in a Pakistani patient. Here, we report a case of acute syphilitic posterior placoid chorioretinopathy in a young Pakistani man.

\section{Case Presentation}

A 25 year-old-male, from outside Karachi, presented to

${ }^{1-3}$ Department of Surgery, 4 Department of Medicine, Aga Khan University Hospital, Karachi, Pakistan.

Correspondence: M. A. Rehman Siddiqui.

Email: rehman.siddiqui@gmail.com us in April 2013 at Shahzad Eye Hospital, Karachi with acute loss of vision in the right eye for two days. There was a history of left eye vision loss six months ago. The patient was otherwise healthy. He was on no regular medications. He was unmarried, and denied any sexual contacts. On examination visual acuity was counting fingers in the right eye, and only perception of light in the left eye. Fundoscopic examination showed vitritis in both eyes, and a flat "placoid" lesion on the right posterior pole (Figure-1). The left eye showed burnt out severe uveitis with swollen disc and vascular sheathing. Furthermore, fundus fluorescein angiography (FFA) revealed extensive inflammation associated with signs of vasculitis and diffuse leakage of capillaries (Figure$2 A \& B)$. Optical coherence tomography showed loss of ellipsoid zone (not shown). On general physical examination, there were maculopapular rashes on the upper back (Figure-1 insert) which raised the suspicion of syphilis. Tests for syphilis including VDRL and FTA-ABS were both positive. Human immuno deficiency virus (HIV) screening was recommended but the patient declined further testing.

He received intravenous penicillin $\mathrm{G}$ and showed signs of improvement. Six week post treatment vision in the right

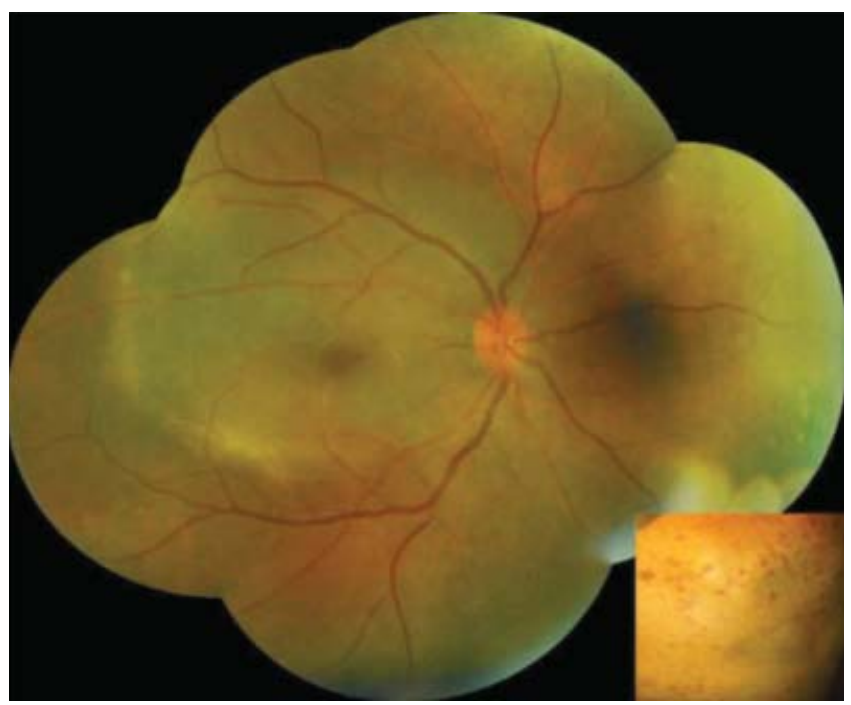

Figure-1: Fundus montage image of right eye showing the placoid lesion temporal to the macula. The skin rashes on the back are clearly visible in the insert. 


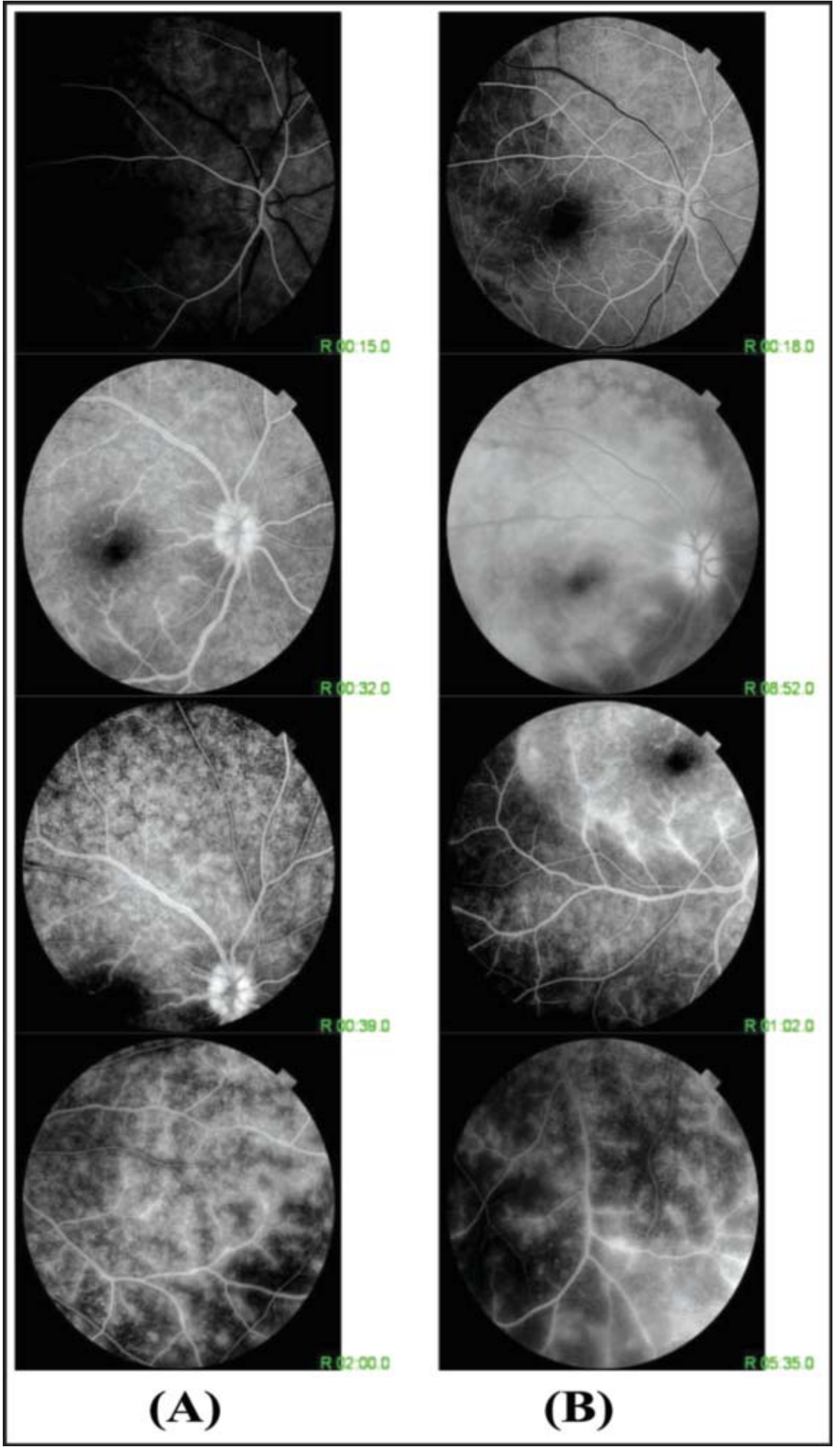

Figure-2: A \& B. various stages of FFA showing extensive inflammation in the eye with signs of vasculitis, and diffuse leakage from capillaries. eye improved to $6 / 18$ and the left improved to $6 / 36$ (Snellen visual acuity, where $6 / 6$ is perfect vision). The Patient's consent was obtained before writing the manuscript.

\section{Discussion}

Syphilis is caused by a gramnegative spirochete Treponema Pallidum. Syphilis is usually classified into four stages: primary, secondary, latent and tertiary. ${ }^{4}$ Ocular complications typically develop in secondary or tertiary syphilis. 2,5 Syphilis may involve one or both eyes; however, bilateral involvement is predominant. Syphilis usually affects people from age 28 to 57 years, ${ }^{6}$ predominantly in men who have sex with men (MSM). ${ }^{5}$ It involves anterior and/or posterior segment or extra ocular nerves as well. It usually takes six weeks to invade the eye, following the systemic infection. In case of nerve invasion, the patient presents with extra-ocular muscle palsies, compromised visual acuity and loss of pupillary light reflex (Argyll Robertson pupil). ${ }^{2}$ The posterior segment is most commonly involved ${ }^{1}$ and the most common finding on posterior segment is uveitis chorioretinitis. ${ }^{2}$ Rarely, uveitis may present as acute syphilitic posterior placoid chorioretinopathy. This term was devised first time in $1990 .{ }^{7}$ In acute syphilitic posterior placoid chorioretinopathy, retinal pigment epithelium in the macula or peri-papillary region gets infected secondary to syphilis. ${ }^{7}$ In one published report, it was reported that auto-immunity plays a significant role in this form of syphilitic uveitis. ${ }^{8}$

Diagnosis of syphilis mainly depends on serology and clinical manifestations. Initial screening is 
done by VDRL and rapid plasma regain (RPR) tests, which should further be confirmed by Treponemal tests (FTA, chemiluminescent micro-particle immunoassay (CMIA) and Treponema pallidum particle agglutination assay (TP-PA) if needed. ${ }^{4}$ Moreover, in case of neurosyphilis, VDRL and FTA tests can also be performed on cerebrospinal fluid, that are highly specific and sensitive respectively. Due to increase in number of co-infection of syphilis with HIV, it is suggested that screening of HIV should be considered in patients with syphilis.9,10 Also, HIV positive serology may result in false negative syphilis test.

Fundus Fluorescein Angiography (FFA) is a particularly useful test in syphilitic uveitis. Findings in syphilis include retinal/capillary leakage, vascular staining, disc hyper fluorescence, and macular edema. In a case report, it was suggested that the finding of Leopard spot on FFA as particularly significant for acute syphilitic posterior placoid chorioretinopathy. ${ }^{7}$ Similarly, the usefulness of spectral domain optical coherence tomography (OCT) has also been described for ocular syphilis. Likewise, one study reported the appearance of SD-OCT in three patients with acute syphilitic posterior placoid chorioretinopathy. ${ }^{11}$ Spectral domain-OCT images showed features which included thickening of the retinal pigment epithelium along with hyper-reflective nodularity, also a break in continuity in photoreceptor inner segment-outer segment junction. Hence, SD-OCT imaging can provide further insight in understanding the pathophysiology of acute syphilitic posterior placoid chorioretinopathy. ${ }^{11}$

Ocular syphilis is usually treated as neurosyphilis. Standard treatment is intra-venous Benzyllpencilline administered continuously for 10-14 days at a dose of 1824 million units (MU) per day.2,3 Corticosteroid may also be given in order to prevent Jarisch-Herxheimer reaction, which could further complicate ocular syphilis. ${ }^{3}$ Similarly, another case report described the possible role of corticosteroids in slowing the progress of acute syphilitic posterior placoid chorioretinopathy; however, complete remission is not possible without antibiotic. ${ }^{8}$ Another treatment option is intramuscular injection of procaine penicillin $G$ one per day along with four times oral probenecid 500 milligrams daily. This regime should continue for 10 to 14 days. $^{2}$ Remission of six cases of syphilitic uveitis have also been reported at Manchester Uveitis Clinic post treatment with intramuscular procaine penicillin $\mathrm{G}$ and oral probenecid. ${ }^{3}$ With prompt antibiotic treatment, prognosis of ocular syphilis is good. ${ }^{2}$

\section{Conclusion}

Cases of syphilis are increasing worldwide approximately at a rate of 12 million every year. Syphilis can involve any structure of the eye. Ocular syphilis should be suspected in all cases of uveitis. Early diagnosis and prompt treatment with appropriate antibiotics can prevent permanent visual damage.

Disclaimer: None to declare.

Conflict of Interest: None to declare.

Funding Disclosure: None to declare.

\section{References}

1. Yang P, Zhang N, Li F, Chen Y, Kijlstra A. Ocular manifestations of syphilitic uveitis in Chinese patients. Retina 2012; 32:1906-14. DOI: 10.1097/IAE.0b013e3182509796.

2. Kiss S, Damico FM, Young LH. Ocular manifestations and treatment of syphilis. Semin Ophthalmol 2005; 20:161-7. DOI: 10.1080/08820530500232092.

3. Doris JP, Saha K, Jones NP, Sukthankar A. Ocular syphilis: the new epidemic. Eye (Lond) 2006; 20:703-5. DOI: 10.1038/sj.eye.6701954

4. Canadian Guidelines on Sexually Transmitted Infections, 2nd ed. Ottawa, Canada: Public Health Agency of Canada, 2006; pp 370.

5. Gaudio PA. Update on ocular syphilis. Curr Opin Ophthalmol 2006; 17:562-6. DOI: 10.1097/ICU.0b013e328010a9b5.

6. Eandi CM, Neri P, Adelman RA, Yannuzzi LA, Cunningham ET Jr. Acute syphilitic posterior placoid chorioretinitis: report of a case series and comprehensive review of the literature. Retina 2012; 32:1915-41. DOI: 10.1097/IAE.0b013e31825f3851.

7. Gass JD, Braunstein RA, Chenoweth RG. Acute syphilitic posterior placoid chorioretinitis. Ophthalmology 1990; 97:1288-97. DOI: 10.1016/s0161-6420(90)32418-1.

8. Ormaechea MS, Hassan M, Nguyen QD, Schlaen A. Acute syphilitic posterior placoid chorioretinopathy: An infectious or autoimmune disease? Am J Ophthalmol Case Rep 2019; 14:70-3. doi: 10.1016/j.ajoc.2019.03.002.

9. Zetola NM, Klausner JD. Syphilis and HIV infection: an update. Clin Infect Dis 2007; 44:1222-8. DOI: 10.1086/513427.

10. Dutta Majumder P, Chen EJ, Shah J, Ching Wen Ho D, Biswas J, See Yin L, et al. Ocular syphilis: an update. Ocul Immunol Inflamm 2019; 27:117-25. doi: 10.1080/09273948.2017.1371765.

11. Burkholder BM, Leung TG, Ostheimer TA, Butler NJ, Thorne JE, Dunn JP. Spectral domain optical coherence tomography findings in acute syphilitic posterior placoid chorioretinitis. J Ophthalmic Inflamm Infect 2014; 4:2. doi: 10.1186/1869-5760-4-2. 